\title{
Optical spectroscopy in CoO: Phononic, electric, and magnetic excitation spectrum within the charge-transfer gap
}

\author{
Ch. Kant, ${ }^{1}$ T. Rudolf, ${ }^{1}$ F. Schrettle, ${ }^{1}$ F. Mayr, ${ }^{1}$ J. Deisenhofer, ${ }^{1}$ P. Lunkenheimer, ${ }^{1}$ M. V. Eremin, ${ }^{1,2}$ and A. Loidl ${ }^{1}$ \\ ${ }^{1}$ Experimental Physics V, Center for Electronic Correlations and Magnetism, University of Augsburg, 86135 Augsburg, Germany \\ ${ }^{2}$ Kazan State University, 420008 Kazan, Russia
}

(Received 5 September 2008; published 3 December 2008)

\begin{abstract}
The reflectivity of single-crystalline $\mathrm{CoO}$ has been studied by optical spectroscopy for wave numbers ranging from 100 to $28000 \mathrm{~cm}^{-1}$ and for temperatures $8<T<325 \mathrm{~K}$. A splitting of the cubic IR-active phonon mode on passing the antiferromagnetic phase transition at $T_{N}=289 \mathrm{~K}$ has been observed. At low temperatures the splitting amounts to $15.0 \mathrm{~cm}^{-1}$. In addition, we studied the splitting of the cubic crystal-field ground state of the $\mathrm{Co}^{2+}$ ions due to spin-orbit coupling, a tetragonal crystal field, and exchange interaction. Below $T_{N}$, magnetic-dipole transitions between the exchange-split levels are identified, and the energy-level scheme can be well described with a spin-orbit coupling $\lambda=151.1 \mathrm{~cm}^{-1}$, an exchange constant $J$ $=17.5 \mathrm{~cm}^{-1}$, and a tetragonal crystal-field parameter $D=-47.8 \mathrm{~cm}^{-1}$. Already in the paramagnetic state electric-quadrupole transitions between the spin-orbit split level have been observed. At high frequencies, two electronic levels of the crystal-field-split $d$ manifold were identified at 8000 and $18500 \mathrm{~cm}^{-1}$.
\end{abstract}

DOI: 10.1103/PhysRevB.78.245103

PACS number(s): 78.30.Am, 63.20.kk, 75.50.Ee, 71.70.Ej

\section{INTRODUCTION}

Strongly correlated transition-metal compounds with partly filled $d$ bands display a variety of properties, interesting for fundamental research and important for future technological applications: Colossal magnetoresistance and multiferroicity in the manganites, high-temperature superconductivity in the cuprates, exotic superconductivity in the ruthenates, and the cobaltites are illuminating examples. The complexity of the ground state is driven by strong electronic correlations and a strong interplay between charge, orbital, spin, and lattice degrees of freedom. In many of these compounds, orbital degrees of freedom play an essential role and access to the orbital state can be obtained by studying the splitting of the $d$ levels, which can reveal the effects of the crystal field (CF), spin-orbit coupling (SOC), and exchange-coupling in the magnetically ordered state. However, experimental methods to study these local $d-d$ excitations are limited. They are electric dipole forbidden and, hence, very weak when compared to Mott-Hubbard $\left(d^{n} d^{n}-d^{n-1} d^{n+1}\right)$ or charge-transfer excitations $\left(d^{n} d^{n}-d^{n+1} L^{-}\right)$, which sometimes are in a similar energy range. Localized $d$ - $d$ excitations have been analyzed using electron-loss spectroscopy ${ }^{1}$ and, recently, with the use of nonresonant inelastic x-ray scattering, Larson et al. ${ }^{2}$ determined the energy scale of $d$ - $d$ excitations within the charge-transfer gaps of $\mathrm{NiO}$ and $\mathrm{CoO}$. The latter results have been quantitatively explained using a local many-body approach by Haverkort et $a l{ }^{3}$ Note that the late transition-metal monoxides are prototypical correlated electron systems and benchmark materials for charge-transfer insulators. ${ }^{4}$

Moreover, transition-metal monoxides are regarded as model systems for spin-phonon coupling effects. The idea of a purely magnetic-order-induced phonon splitting has been put forth by Massidda et $a .^{5}$ for the antiferromagnetic (AFM) transition-metal monoxides and has been further substantiated in a recent work by Luo et al. ${ }^{6}$ The splitting of the transverse optic modes below the Néel temperature $T_{N}$ has indeed been experimentally documented by Chung et $\mathrm{l.}^{7}$ in $\mathrm{MnO}$ and $\mathrm{NiO}$ by inelastic neutron scattering and by Rudolf et $\mathrm{al}^{8}{ }^{8}$ in $\mathrm{MnO}$ by far-infrared (FIR) spectroscopy. The splitting of phonon modes has also been observed in a number of spinel compounds at the onset of AFM order ${ }^{9-11}$ and has been interpreted in terms of a spin-driven Jahn-Teller (JT) effect. ${ }^{12,13}$

The purpose of this study is to reinvestigate the optical properties of $\mathrm{CoO}$ by infrared (IR) spectroscopy with regard to spin-phonon coupling effects and $d-d$ excitations. $\mathrm{CoO}$ has first been synthesized by Klemm and Schüth. ${ }^{14}$ Magneticsusceptibility measurements ${ }^{15-19}$ show an antiferromagnetic transition at approximately $290 \mathrm{~K}$ with a negative CurieWeiss temperature of the order of the AFM transition temperature. The onset of magnetic order is accompanied by a structural phase transition with a small tetragonal distortion. ${ }^{20}$ At room-temperature $\mathrm{CoO}$ is paramagnetic and exhibits the cubic $\mathrm{NaCl}$ structure (space group $F m \overline{3} m$; a $=0.42495 \mathrm{~nm}$ ) while at $92 \mathrm{~K}$ the lattice parameters of the tetragonal cell are $a=0.42552 \mathrm{~nm}$ and $c / a=0.9884$. Later on, low-temperature $\mathrm{x}$-ray diffraction experiments revealed a rhombohedral distortion in addition to the tetragonal distortion, ${ }^{21}$ while high-resolution synchrotron powder diffraction even manifested a monoclinic symmetry (space group $\mathrm{C} 2 / \mathrm{m}$ ) of antiferromagnetic $\mathrm{CoO} .{ }^{22}$ The spin structure of $\mathrm{CoO}$ in the AFM state has been determined by neutronscattering diffraction by Shull et al. ${ }^{23}$ On the basis of these data, it has been concluded that the magnetic moments in $\mathrm{CoO}$ are arranged in ferromagnetic (111) planes with the preferred spin direction along [ $\overline{1} \overline{1} 7]$, which is intermediate to the (111) plane and the tetragonal axis. ${ }^{24}$ However, there has been some dispute about the true magnetic structure and possible multispin configurations occurring in $\mathrm{CoO}^{25-28} \mathrm{Al}-$ though previous absorption measurements reported the observation of $d-d$ excitations in the paramagnetic (PM) as well as in the AFM state, ${ }^{29-32}$ a comprehensive and unambiguous description of the splittings and determination of the relevant interaction parameters is still missing. We are able to de- 
scribe the observed splittings in the PM and antiferromagnetically ordered state very well by taking into account SOC, tetragonal crystal field, and exchange splitting contributions.

\section{EXPERIMENTAL DETAILS AND SAMPLE CHARACTERIZATION}

Single crystals polished to optical quality (space group $F m \overline{3} m, a=0.425 \mathrm{~nm}$ at room temperature) in the form of platelets with dimensions of approximately $1 \mathrm{~cm}^{2}$ and $1 \mathrm{~mm}$ thickness were purchased from MaTecK GmbH. Impurities such as $\mathrm{Fe}$ or Ni were less than $0.01 \%$. For characterization, the magnetic properties were studied using a commercial superconducting quantum interference device magnetometer (Quantum Design MPMS-5) with external magnetic fields up to $50 \mathrm{kOe}$. The heat capacity was measured in a Quantum Design physical properties measurement system for temperatures from $2<T<300 \mathrm{~K}$. The dielectric properties were determined using a frequency-response analyzer (Novocontrol) at frequencies between $1 \mathrm{~Hz}$ and $1.5 \mathrm{MHz}{ }^{33}$ For these measurements silver-paint contacts were applied to opposite sides of the platelets. The reflectivity measurements were carried out using the Bruker Fourier-transform spectrometers IFS $113 \mathrm{v}$ and IFS $66 \mathrm{v} / \mathrm{S}$, which both are equipped with a He-flow (4-600 K) cryostat. Using different light sources, different beam splitters and different detectors, we were able to cover the frequency range from 100 to $28000 \mathrm{~cm}^{-1}$. For the analysis of our reflectivity spectra, we derived the complex dielectric constant or the complex index of refraction by means of Kramers-Kronig transformation with a constant extrapolation toward low frequencies and a smooth power law extrapolation at high wave numbers.

It is known from previous experiments and publications that even high-quality single crystals of $\mathrm{CoO}$ can suffer from the intergrowth of $\mathrm{Co}_{3} \mathrm{O}_{4}$ clusters. It was shown that small impurity-free single crystals could only be obtained by annealing $\mathrm{CoO}$ crystals in Co vapor (see Ref. 19). Therefore, we characterized our samples carefully by measuring the magnetic susceptibility, the specific heat, and the dielectric properties. Despite the fact that $\mathrm{CoO}$ is a thoroughly studied transition-metal oxide, we found that the information regarding these basic properties is incomplete; the temperature dependence of the magnetic susceptibility of $\mathrm{CoO}$ has been studied by Singer ${ }^{16}$ between 100 and $800 \mathrm{~K}$. The broad temperature range of this investigation allowed a rather precise determination of the Curie-Weiss temperature $(-330 \mathrm{~K})$ and paramagnetic moment $\left(5.25 \mu_{B}\right)$. A careful susceptibility analysis has been reported in Ref. 19. These authors removed nonstoichiometries by heating a small single crystal in Co vapor and also performed measurements under external stress conditions. We are not aware of any detailed investigation of the heat capacity specifically down to low temperatures. Around $T_{N}$, it has been investigated in Refs. 34 and 35 the specific heat over a broader temperature range, namely from $100<T<500 \mathrm{~K}$ is documented in Refs. 36-38. The dielectric permittivity has been published by Rao and Smakula ${ }^{39}$ measuring the dielectric constant and the dielectric loss of $\mathrm{CoO}$ for frequencies between $100 \mathrm{~Hz}$ and $1 \mathrm{MHz}$ from liquid nitrogen up to room temperature.

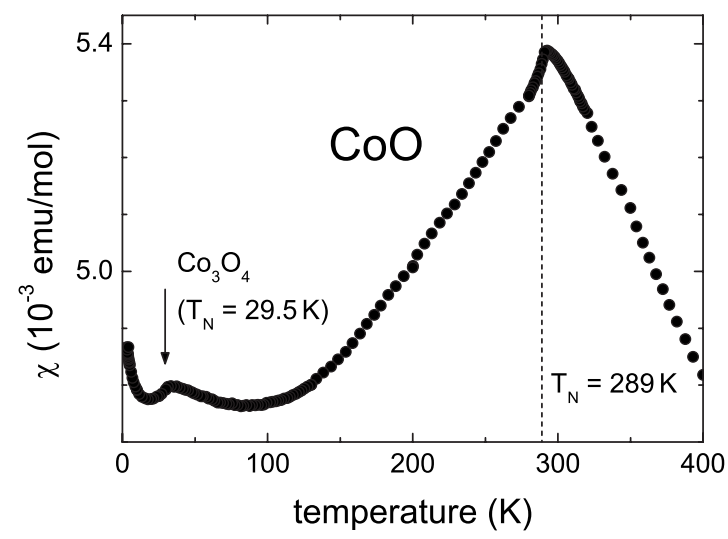

FIG. 1. Temperature-dependent magnetic susceptibility of a single-crystalline $\mathrm{CoO}$ platelet, with the external magnetic field directed perpendicular to the (111) plane. The measurement was performed at an external magnetic field $\mu_{0} H=0.1 \mathrm{~T}$. The magnetic ordering temperature of the impurity phase $\mathrm{Co}_{3} \mathrm{O}_{4}$ is indicated by an arrow.

Figure 1 shows the temperature dependence of the magnetic susceptibility as measured in an external field of $\mu_{0} H$ $=0.1 \mathrm{~T}$ for temperatures $2.5<T<400 \mathrm{~K}$, which is in good agreement with previous publications. The AFM transition appears as a sharp cusp close to $290 \mathrm{~K}$. Below the ordering temperature, the susceptibility decreases before a slight cusp becomes visible close to $30 \mathrm{~K}$, which corresponds to AFM ordering temperature $T_{N}=29.5 \mathrm{~K}_{\text {of }} \mathrm{Co}_{3} \mathrm{O}_{4}{ }^{40}$ Toward lower temperatures we observe an increase below about $20 \mathrm{~K}$ which signals a Curie contribution due to free Co spins probably located in grain boundaries or domain walls. Our susceptibility data in the range $350<T<400 \mathrm{~K}$ yields a CurieWeiss temperature of approximately $-450 \mathrm{~K}$ and an effective moment $p_{\text {eff }}=5.7 \mu_{B}$, which have to be compared to $\Theta_{\mathrm{CW}}=-330 \mathrm{~K}$ and $p_{\text {eff }}=5.25 \mu_{B}$ obtained by Singer. ${ }^{16}$ The discrepancy is attributed to the larger temperature range in the PM phase up to $800 \mathrm{~K}$ in the latter study, which naturally leads to more reliable parameters. Assuming a spin-only contribution of high-spin $\mathrm{Co}^{2+}$-ions with $S=3 / 2$, the effective moment $p_{\text {eff }}=5.25 \mu_{B}$ results in an effective $g$ value $g=2.71$, which indicates a non-negligible contribution of the orbital momentum. ${ }^{41,42}$

The temperature dependence of the molar heat capacity $c_{p}$ is documented in Fig. 2. The AFM transition can clearly be seen. From a closer inspection of the anomaly at the AFM ordering we determine a phase-transition temperature $T_{N}$ $=289 \mathrm{~K}$ in agreement with our susceptibility data. At room temperature, just above $T_{N}$, the heat capacity amounts approximately to $6.5 R$ and is significantly enhanced when compared to a solid with six phonon branches, i.e., 2 $\times 3$ degrees of freedom. This enhanced heat capacity likely results from crystal-field contributions or spin fluctuations. It should be mentioned also that Dulong-Petit's law actually addresses the specific heat measured at constant volume and not at constant pressure, but such corrections can often be neglected for solid states.

At low temperatures the heat capacity can best be fit utilizing a $T^{3}$ law and in addition a small linear term. A fit taking into account only data below $12 \mathrm{~K}$ results in a Debye 


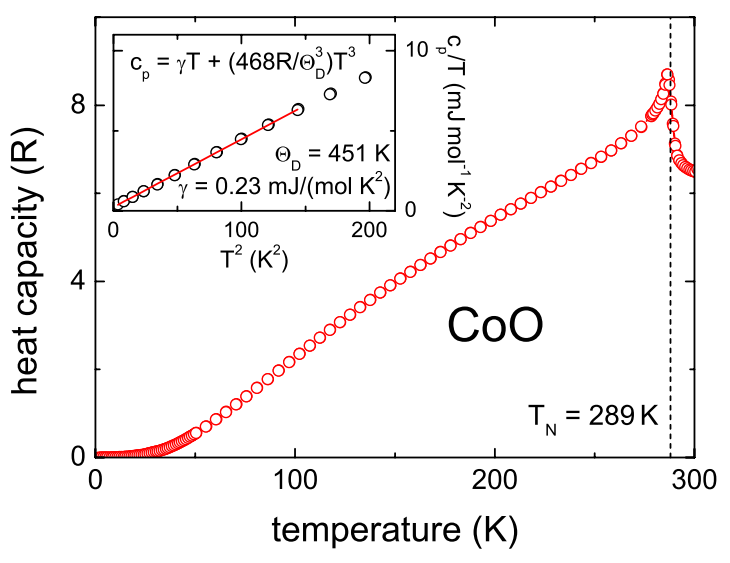

FIG. 2. (Color online) Heat capacity of $\mathrm{CoO}$ vs temperature. Inset: plot of $c_{p} / T$ vs $T^{2}$ (see text). The best fit of the lowtemperature specific heat is achieved taking into account a small linear term $\gamma=0.23 \mathrm{~mJ} /\left(\mathrm{mol} \mathrm{K}^{2}\right)$ (solid line in inset).

temperature of $\Theta_{D}=451 \mathrm{~K}$ (see inset of Fig. 2), which is expected from the phonon dynamics. From the normal modes of vibrations Kushwaha ${ }^{43}$ calculated $\Theta_{D}(T)$. He found $\Theta_{D} \approx 500 \mathrm{~K}$ toward $0 \mathrm{~K}$ and values approaching $600 \mathrm{~K}$ at the Néel temperature, the latter being in good agreement with published experimental results. ${ }^{44}$ Hence, at low temperatures magnetic contributions seem to play only a minor role in CoO. Obviously, the AFM magnons display a large gap due to strong SOC, which would explain the absence of any dispersive magnon contributions at low temperatures and the enhanced heat capacity at higher temperatures when compared to a nonmagnetic solid. This could also explain the small linear term (see inset in Fig. 2). To strengthen these arguments a more detailed analysis including the heat capacity of phonons, magnons, and Schottky-type crystal field levels is necessary but beyond the scope of this paper.

Finally, we also measured the dielectric constant $\varepsilon^{\prime}$ and the conductivity $\sigma^{\prime}$ of $\mathrm{CoO}$ between 4 and $500 \mathrm{~K}$ and 1 $\mathrm{Hz}-1.5 \mathrm{MHz}$. The results are documented in Fig. 3. Only below room temperature and for high measuring frequencies $f$ the static dielectric constant is approached, yielding approximately $\varepsilon_{s}=13$ at low temperatures [Fig. 3(a)]. At high temperatures the real part of the dielectric constant is dominated by ac contributions. It reaches colossal values up to $10^{4}$ (not shown), which may be due to hopping conductivity ${ }^{45}$ or Maxwell-Wagner polarization. ${ }^{46}$ Only for $f=1.5 \mathrm{MHz}$ the room-temperature value of the static dielectric constant $\varepsilon_{s}=14.2$ can be estimated. This value is slightly enhanced when compared to published results by Rao and Smakula, ${ }^{39}$ who found $\varepsilon_{s}=12.9$ at room temperature. At relatively high measuring frequencies, the AFM ordering is displayed by a small cusp in the temperature-dependent dielectric constant [inset of Fig. 3(a)].

The conductivity $\sigma^{\prime}$ of $\mathrm{CoO}$ is shown in Fig. 3(b). Here frequency independent $\mathrm{dc}$ conductivity dominates above room temperature, while ac contributions dominate at lower temperatures. A closer inspection of the frequency dependence of the conductivity close to $100 \mathrm{~K}$ in the Fig. 3(b) reveals that it deviates from a linear behavior and can best be represented by a power law, $\sigma \sim \omega^{s}$, with $s \sim 0.7$, a typical

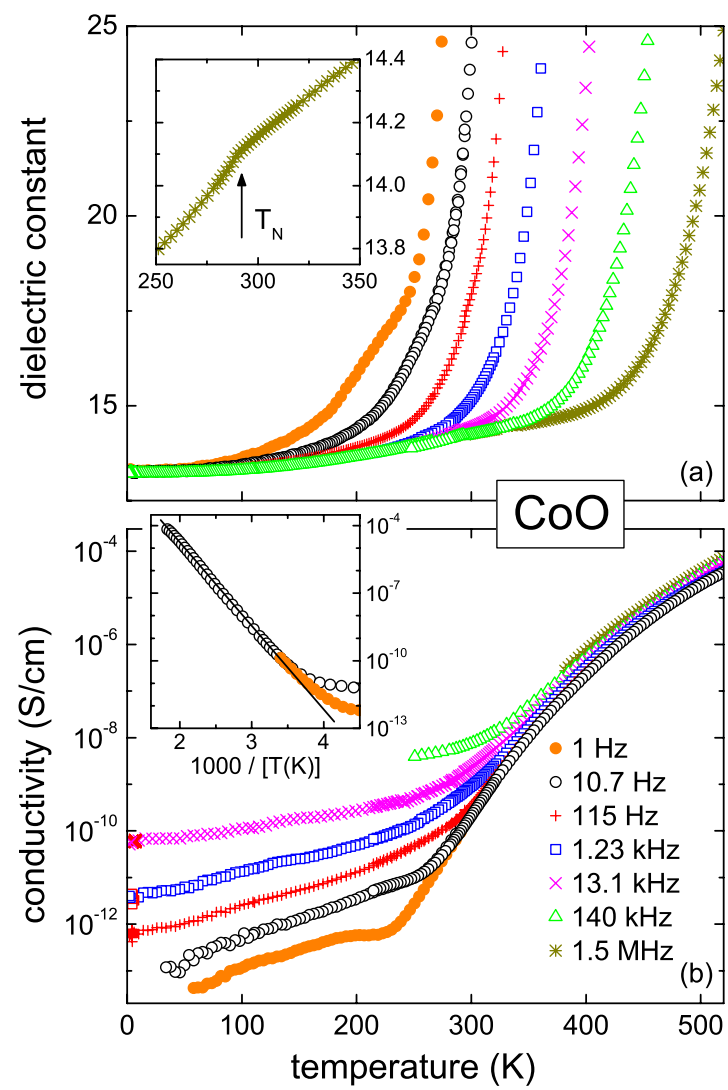

FIG. 3. (Color online) Temperature dependence of the real part of the dielectric constant $\varepsilon^{\prime}$ [upper frame: (a)] and real part of the conductivity $\sigma^{\prime}$ [lower frame: (b)] of $\mathrm{CoO}$ measured at different frequencies between $1 \mathrm{~Hz}$ and $1.5 \mathrm{MHz}$. A (100) platelet has been investigated with the electrical field perpendicular to the (100) plane. The inset in (a) demonstrates the presence of an anomaly in $\varepsilon^{\prime}(1.5 \mathrm{MHz}) \approx \varepsilon_{s}$ at the magnetic phase transition. The inset in (b) shows the temperature dependence of the dc conductivity in an Arrhenius-type presentation. The line corresponds to a band gap of $1.5 \mathrm{eV}$.

signature of hopping conduction in disordered solids. ${ }^{45}$ Similar behavior was found in numerous transition-metal oxides (see, e.g., Ref. 47). The inset of Fig. 3(b) shows the dc contributions at high temperatures in an Arrhenius-type representation. The conductivity can well be described assuming a gap of approximately $1.5 \mathrm{eV}$, which compares well with published results. ${ }^{39}$

\section{MODEL CALCULATIONS AND ANALYSIS}

The reflectivity $R(\omega)$ of an interface is governed by Fresnel's equations. In case of light impinging on a sample surface at normal incident, they can be simplified according to

$$
R(\omega)=r(\omega) r^{*}(\omega)=\left|\frac{\sqrt{\varepsilon(\omega)}-\sqrt{\mu(\omega)}}{\sqrt{\varepsilon(\omega)}+\sqrt{\mu(\omega)}}\right|^{2} .
$$

Here, $r$ is the complex reflectance coefficient, $\varepsilon$ is the dielectric function, and $\mu$ is the magnetic permeability. In most compounds, $\mu$ has negligible influence on the reflectivity 
spectrum and is usually set to unity in the optical frequency range. Equation (1) is valid for all isotropic, homogeneous, local, and linear materials in the limit of classical electrodynamics.

In order to analyze our reflectivity data, we use model functions for both the dielectric function and the magnetic permeability. The phononic and electronic contributions can be obtained by the factorized dielectric function ${ }^{48}$

$$
\varepsilon(\omega)=\varepsilon_{\infty} \prod_{j} \frac{\omega_{\mathrm{LO} j}^{2}-\omega^{2}-i \gamma_{\mathrm{LO} j} \omega}{\omega_{\mathrm{TO} j}^{2}-\omega^{2}-i \gamma_{\mathrm{TO} j} \omega},
$$

where, in case of the phonon modes, $\omega_{\mathrm{TO} j}, \omega_{\mathrm{LO} j}, \gamma_{\mathrm{TO} j}$, and $\gamma_{\mathrm{LO} j}$ can be directly interpreted as eigenfrequencies $\left(\omega_{j}\right)$ and damping constants $\left(\gamma_{j}\right)$ of the transversal (TO) and longitudinal (LO) optical modes, respectively. $j$ is an index variable which runs over all phononic and electronic excitations. $\varepsilon_{\infty}$ arises from high-frequency electronic absorption processes beyond the phonon domain. The dielectric strength $\Delta \varepsilon_{j}$ of excitation $j$ can explicitly be derived from the parameters of the model function if the resonances are well separated

$$
\Delta \varepsilon_{j}=\varepsilon_{\infty} \frac{\omega_{\mathrm{LO} j}^{2}-\omega_{\mathrm{TO} j}^{2}}{\omega_{\mathrm{TO} j}^{2}} \prod_{i \geq j+1} \frac{\omega_{\mathrm{LO} i}^{2}}{\omega_{\mathrm{TO} i}^{2}} .
$$

The effective ionic plasma frequency $\Omega_{j}$ of each excitation can then be expressed by

$$
\Omega_{j}^{2}=\Delta \varepsilon_{j} \omega_{\mathrm{TO} j}^{2}
$$

An alternative way to model the dielectric function is by utilizing a sum of Lorentz oscillators

$$
\varepsilon(\omega)=\varepsilon_{\infty}+\sum_{j} \frac{\Omega_{j}^{2}}{\omega_{j}^{2}-\omega^{2}-i \gamma_{j} \omega} .
$$

Here, three parameters are adjustable per mode: $\omega_{j}$ and $\gamma_{j}$ are eigenfrequency and damping of the $j$ th resonance, respectively. The third fit parameter, which enters Eq. (5), is the plasma frequency $\Omega_{j}$ of mode $j$. Comparing the model dielectric functions one can deduce that Eqs. (2) and (5) become identical if the damping coefficients of $\mathrm{TO}$ and $\mathrm{LO}$ modes are equal.

It was shown by $\mathrm{Scott}^{49}$ that the following equation holds true for the overall plasma frequency in a multimode system:

$$
\Omega^{2}=\sum_{k} \Omega_{k}^{2}=\frac{\varepsilon_{\infty}}{V \varepsilon_{\mathrm{vac}}} \sum_{l} \frac{\left(Z_{l}^{*} e\right)^{2}}{m_{l}} .
$$

$V$ denotes the unit-cell volume, and $Z_{l}^{*} e$ is the effective charge of the $l$ th ion with mass $m_{l}$ contributing to a specific phonon mode. $\varepsilon_{\mathrm{vac}}$ is the dielectric permittivity of free space.

Our reflectivity data have been analyzed by utilizing Eqs. (1), (2), and (5) and a fit routine which was developed by Kuzmenko. ${ }^{50}$

We also studied phonon eigenfrequencies and damping coefficients as a function of temperature $T$. To account for purely anharmonic effects, we assume

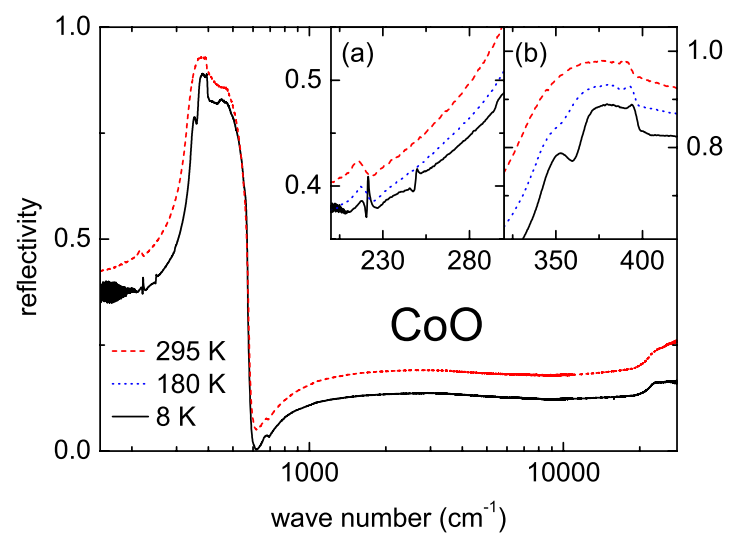

FIG. 4. (Color online) Reflectivity of $\mathrm{CoO}$ between 150 and $28000 \mathrm{~cm}^{-1}$ at three different temperatures. The curves in the main frame and inset (b) are successively shifted by an amount of 0.05 for clarity. Inset (a) shows the frequency regime between 200 and $300 \mathrm{~cm}^{-1}$ on an expanded scale, while inset (b) provides a closer look in the frequency range between 320 and $420 \mathrm{~cm}^{-1}$, where the splitting of the phonon mode occurs.

$$
\omega_{\mathrm{TO} j}(T)=\omega_{0 j}\left(1-\frac{c_{j}}{\exp \left(\Theta_{D} / T\right)-1}\right)
$$

for the temperature dependence of the transverse eigenfrequency $\omega_{\mathrm{TO} j}$ and

$$
\gamma_{\mathrm{TO} j}(T)=\gamma_{0 j}\left(1+\frac{d_{j}}{\exp \left(\Theta_{D} / T\right)-1}\right)
$$

for the temperature dependence of the damping $\gamma_{\mathrm{TO} j}$ of mode $j$, respectively. $\omega_{0 j}$ and $\gamma_{0 j}$ are the eigenfrequency and damping of mode $j$ at $0 \mathrm{~K}$. $\Theta_{D}$ denotes the Debye temperature and was treated as a free fitting parameter as well as the constants $c_{j}$ and $d_{j}$, which determine the strength of the anharmonic contributions. Detailed calculations of temperature and frequency-dependent anharmonicity can be found in Ref. 51.

\section{RESULTS AND DISCUSSION}

Figure 4 presents the reflectivity of $\mathrm{CoO}$ between 150 and $28000 \mathrm{~cm}^{-1}$ for different temperatures above and below the antiferromagnetic ordering temperature $\left(T_{N}=289 \mathrm{~K}\right)$. At room temperature the reflectivity is dominated by a broad reststrahlen band between 300 and $600 \mathrm{~cm}^{-1}$, which is due to the optical phonons of the $\mathrm{NaCl}$-like structure. At zero wave vector the two transverse phonons are degenerate and their eigenfrequencies are determined by the strong increase in the reflectivity close to $300 \mathrm{~cm}^{-1}$. The frequency of the longitudinal optical phonon is determined by the steep decrease in the reflectivity at $600 \mathrm{~cm}^{-1}$. Ideally, the reflectivity in between these two characteristic frequencies, where $\varepsilon^{\prime}$ is negative, should be close to unity, at least at low temperatures where anharmonic effects are small. Deviations from this idealized behavior and additional structures usually result from multiple phonon excitations, which will become more pronounced with increasing temperature. The observed structure in the reflectivity on top of the reststrahlen band around $400 \mathrm{~cm}^{-1}$ could result from such a two-phonon pro- 
cess involving zone boundary optical and acoustical modes, which sum up to a zero wave-vector excitation with a dipole moment transferred from the transverse optic phonon mode. Indeed, a weak multiphonon structure close to $400 \mathrm{~cm}^{-1}$ has been calculated by Upadhyaya and Singh. ${ }^{52}$ However, much stronger peaks in the combined density of states are calculated to appear close to 435, 500, and $535 \mathrm{~cm}^{-1}$, which could not be identified unambiguously. In addition, it has to be stated that the temperature dependence of the observed structures [inset (b) of Fig. 4] is not in accord with anharmonic effects. Multiphonon structures usually become more significant on increasing temperatures, a fact that definitely is not observed in $\mathrm{CoO}$. The inset (b) of Fig. 4 documents that the $400 \mathrm{~cm}^{-1}$ anomaly remains almost constant from the lowest to the highest temperatures. Hence, the origin of this structure close to $400 \mathrm{~cm}^{-1}$ remains unsettled. In addition to the reststrahlen band due to the phonons, an increase in the reflectivity close to $22000 \mathrm{~cm}^{-1}$ signals electronic interband transitions.

At first sight, small additional anomalies close to 220 and $700 \mathrm{~cm}^{-1}$ can be seen in the frequency-dependent roomtemperature reflectivity displayed in Fig. 4. On cooling and passing the AFM phase transition no significant shifts or changes are visible, but the appearance of small additional bands close to 250 and $350 \mathrm{~cm}^{-1}$ can be detected [see insets (a) and (b) of Fig. 4]. Inset of Fig. 4(a) also documents that the rather broad feature of the $220 \mathrm{~cm}^{-1}$ transition is superimposed by a sharp structure when entering the magnetically ordered phase. The anomaly close to $249 \mathrm{~cm}^{-1}$ exhibits a similar shape, namely a dip followed by a peak. Note that weak features also appear at low temperatures at 142 and $295 \mathrm{~cm}^{-1}$ as shown in Fig. 5. The interference fringes at low frequencies are due to the finite thickness of the plan-parallel sample. At this point we would like to state that the anomalies at $142,220,249,295$, and close to $700 \mathrm{~cm}^{-1}$ are of electronic origin and will be discussed later. The additional band close to $350 \mathrm{~cm}^{-1}$, which evolves just below the magnetic ordering [see inset (b) of Fig. 4], represents the magnetic-order induced phonon splitting.

We converted the reflectivity spectrum into a frequencydependent extinction coefficient $\kappa$, which is the imaginary part of the refractive index, in order to reveal the transition features more clearly. The reststrahlen band now roughly corresponds to a loss peak (upper panel of Fig. 5). It splits at low temperatures which becomes nicely visible as a clear double peak structure. An enlarged view of the anomalies between 200 and $300 \mathrm{~cm}^{-1}$ is provided in inset (a). Again, at room temperature $\left(T>T_{N}\right)$, only one single peak is seen, which we denote as $Q_{1}$ in the following. It becomes superimposed by an additional negative cusp at low temperatures. The detailed temperature evolution is shown in the lower panel of Fig. 5. In the following, we label the three negative cusps at 221,249 , and $295 \mathrm{~cm}^{-1}$ with $M_{2}-M_{4}$, respectively. These excitations appear below $100 \mathrm{~K}$ only, deep in the magnetically ordered state. They increase slightly in intensity and saturate below $25 \mathrm{~K}$, but show no significant shifts in frequency. The inset in the lower panel documents the occurrence of a further excitation $\left(M_{1}\right)$ at $142 \mathrm{~cm}^{-1}$. It behaves similar to $M_{2}-M_{4}$ as it is diplike and vanishes at approximately $100 \mathrm{~K} . M_{1}$ strongly resembles the results of infrared
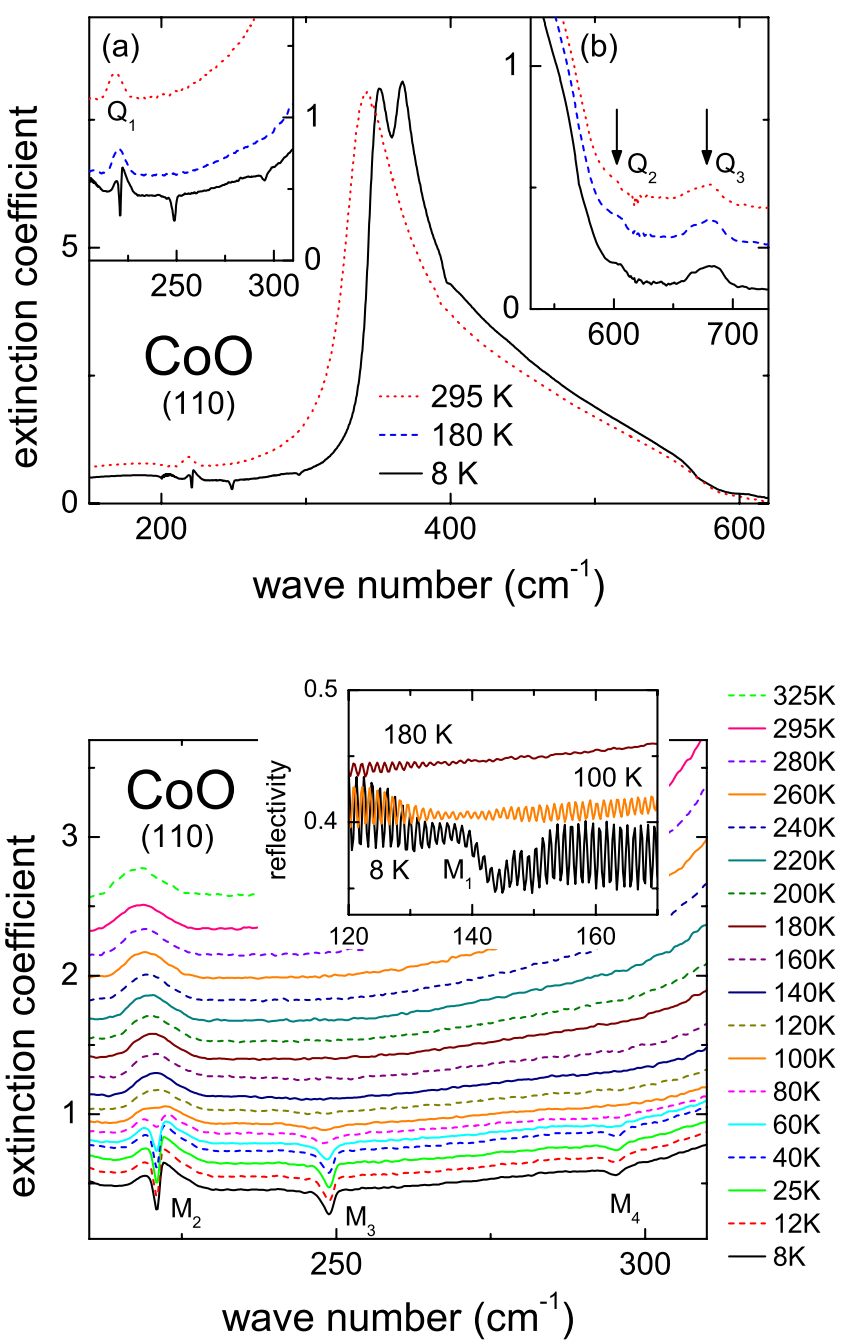

FIG. 5. (Color online) Upper panel: extinction coefficient $\kappa$ vs wave numbers at 8 and $295 \mathrm{~K}$. The insets show enlarged regions at $\left(205-310 \mathrm{~cm}^{-1}\right)$ and $\left(530-730 \mathrm{~cm}^{-1}\right)$ wave numbers for $\kappa$ at 8 , 180 , and $295 \mathrm{~K}$. The curves in both insets are separated by an amount of 0.2 for clarity. Lower panel: sequence of temperaturedependent measurements of the extinction coefficient vs wave numbers between 210 and $320 \mathrm{~cm}^{-1}$ and for temperatures $8<T$ $<325 \mathrm{~K}$. The data are cumulatively shifted by an amount of 0.1 for clarity. An electric transition close to $220 \mathrm{~cm}^{-1}$ is visible already at temperatures above the magnetic phase transition $\left(T_{N}=289 \mathrm{~K}\right)$. At $100 \mathrm{~K}$ magnetic-dipole transitions evolve, which gain strength on decreasing temperature. The inset shows an expanded scale of the reflectivity between 120 and $170 \mathrm{~cm}^{-1}$ to demonstrate the appearance of a further excitation close to $142 \mathrm{~cm}^{-1}$ (see text).

absorption by Milward, ${ }^{30}$ who detected a strong absorption at $142.3 \mathrm{~cm}^{-1}$. Similar findings were revealed in the Raman studies of Hayes and Perry. ${ }^{53}$ This is an experimental evidence that $M_{1}-M_{4}$ are intrinsic excitations of $\mathrm{CoO}$ since they appear at approximately at the same temperature and show no spectral changes at the ordering temperature of the impurity phase $\mathrm{Co}_{3} \mathrm{O}_{4}$. We further see small kinks at 600 and $680 \mathrm{~cm}^{-1}$ [inset (b) of Fig. 5], which we will denote as $Q_{2}$ and $Q_{3}$, respectively. They reveal almost no temperature dependence and do not coincide with calculated multiphonon bands. ${ }^{52}$ 


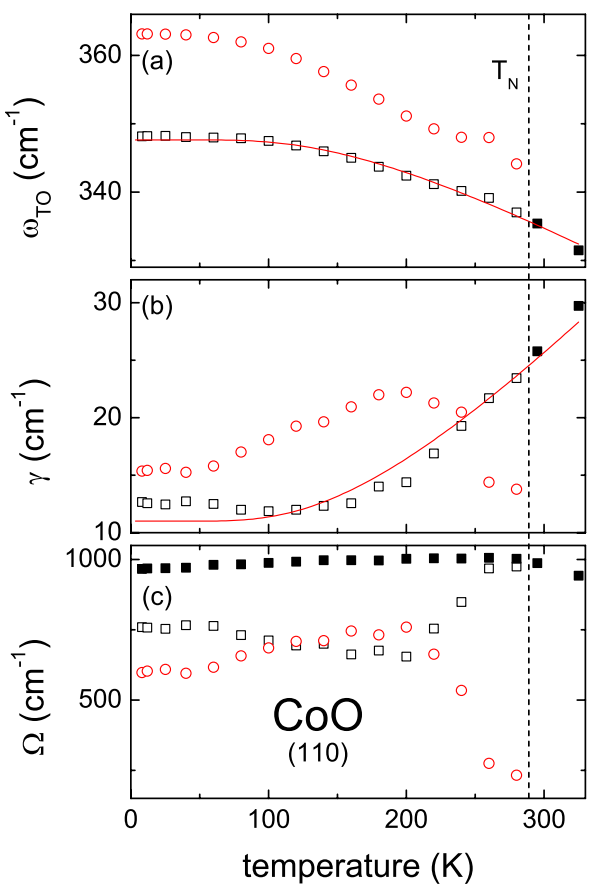

FIG. 6. (Color online) Temperature dependencies of (a) eigenfrequency, (b) damping constants, and (c) effective ionic plasma frequencies of the transverse optical phonon modes of $\mathrm{CoO}$. The main mode (empty squares) and the split-off mode, which appears in the antiferromagnetic phase (empty circles), are shown. All measurements have been performed with single-crystalline platelets with the (110) surface close to normal incidence. The solid lines in (a) and (b) were calculated assuming a simple anharmonic model [see Eqs. (7) and (8)]. Black solid squares denote the respective quantity above $T_{N}$ or in (c) the overall plasma frequency.

In the following we will first discuss the phonon excitations before we turn to the nature of the electronic excitations occurring below and above the Néel temperature.

\section{A. Phonon excitations}

We tried to fit the reflectivity spectra using a fourparameter fit, but the anomalies on top of the reststrahlen band together with the splitting of the phonon modes below $T_{N}$ did not allow for a satisfactory fitting procedure. Therefore, we transformed the reflectivity into dielectric function and fitted these spectra using a Lorentz fit with three parameters as outlined in Eq. (5). For comparison, we performed four-parameter fits in the paramagnetic phase and found good agreement between the two procedures. The results of the Lorentz fits are plotted in Fig. 6. The upper frame (a) gives the temperature dependence of the TO eigenfrequencies. Assuming that the main distortion occurring below $T_{N}$ is tetragonal, ${ }^{20}$ the triply degenerate $T_{1 u}$ of the cubic rocksalt structure at room temperature should split into a doublet and a singlet. Such a scenario is in agreement with the observed splitting. The mode with the larger spectral weight at high temperatures smoothly evolves from the cubic roomtemperature phase and increases from $335.4 \mathrm{~cm}^{-1}$ at room temperature to $348.1 \mathrm{~cm}^{-1}$ at liquid He temperatures. Below $T_{N}$ a second mode splits off with a higher eigenfrequency and increases up to $363.1 \mathrm{~cm}^{-1}$ at the lowest temperature. Note that the abrupt appearance of the second phonon mode in Fig. 6(a) is probably a result of the fitting procedure. Only below $240 \mathrm{~K}$ a reliable fit with two-phonon excitations was possible. It is reasonable to assume that the splitting is a continuous process as it was observed for $\mathrm{MnO}^{8}$ At low temperatures the splitting of the $\mathrm{TO}$ modes in $\mathrm{CoO}$ amounts $15.0 \mathrm{~cm}^{-1}$. We would like to recall that the low-temperature magnetic phase reveals a monoclinic symmetry ${ }^{54}$ and one certainly can expect a large number of IR active phonons. However, these additional splittings are probably below the experimental resolution $\left(<\sim 0.5 \mathrm{~cm}^{-1}\right)$. In comparison to $\mathrm{MnO}$, where the overall splitting of the TO mode in the antiferromagnetic state was estimated to be approximately $30 \mathrm{~cm}^{-1,8}$ the splitting is reduced by a factor of 2 . Since $\Theta_{\mathrm{CW}} / T_{N} \sim 1$ in $\mathrm{CoO}$, one may infer that spin frustration indeed may account for the large magnetic-order induced phonon splitting in $\mathrm{MnO}$.

The temperature dependence of the damping of the transverse phonon modes is shown in Fig. 6(b). The damping of the cubic $T_{1 u}$ continuously decreases toward low temperatures and evolves again smoothly into the mode with larger spectral weight below $T_{N}$ and reaches approximately $12.5 \mathrm{~cm}^{-1}$ at low temperatures. Such a behavior is expected for an anharmonic solid, and both the temperature dependences of the eigenfrequency and the damping are well described by fits [solid lines in Figs. 6(a) and 6(b)] according to Eqs. (7) and (8), respectively. The fit yields a Debye temperature of $\Theta_{D}=526 \mathrm{~K}$, an enhanced value compared to the low-temperature thermodynamic Debye temperature (see Fig. 2) but comparable to the experimentally determined Debye temperatures at ambient conditions. ${ }^{4}$ The damping of the second mode below $T_{N}$ cannot be described by a simple anharmonic behavior.

The temperature dependence of the ionic plasma frequency of the optical modes is shown in Fig. 6(c). Both modes roughly are of equal strength with a value of $700 \mathrm{~cm}^{-1}$ from lowest temperatures up to $200 \mathrm{~K}$. On further approaching the phase boundary, the main mode gains weight reaching a value of $990 \mathrm{~cm}^{-1}$, whereas the split-off mode rapidly gets suppressed. Due to the overlap of the two modes and the decreasing weight of the second mode, the values above $200 \mathrm{~K}$ contain a larger uncertainty.

Finally, the eigenfrequencies and damping constants of the TO and LO modes have also been obtained from a fourparameter fit at room temperature, where only the cubic $T_{1 u}$ mode is observable. The TO and $\mathrm{LO}$ eigenfrequencies were determined as 335.7 and $562.1 \mathrm{~cm}^{-1}$, respectively. The former value coincides with the one obtained from the Lorentz fit as expected. In Table I these values are compared to the ones reported earlier by Gielisse et al..$^{55}$ and Sakurai et $a l .{ }^{56}$ The overall agreement seems satisfactory even though the eigenfrequency of the LO mode of the neutron-scattering study is off by almost $8 \%$ when compared to our result. However, no longitudinal optical eigenfrequency was reported at room temperature. It should be mentioned that based on the experimental phonon excitations, the phonon dynamics of $\mathrm{CoO}$ has been calculated using lattice-dynamic models of different complexity ${ }^{43,52,57}$ reaching satisfactory agreement between experimental data and model calcula- 
TABLE I. Phonon excitations in $\mathrm{CoO}$ observed in the present work, compared to reports in literature. All eigenfrequencies are given in $\mathrm{cm}^{-1}$.

\begin{tabular}{ccccc}
\hline \hline \multicolumn{2}{c}{ FIR } & & \multicolumn{2}{c}{ Neutron scattering } \\
\hline \multicolumn{2}{c}{ (This work) } & Ref. 55 & \multicolumn{2}{c}{ Ref. 56 } \\
\hline $8 \mathrm{~K}$ & $295 \mathrm{~K}$ & $295 \mathrm{~K}$ & $110 \mathrm{~K}$ & $425 \mathrm{~K}$ \\
$348.1(\mathrm{TO} 1)$ & $335.7(\mathrm{TO})$ & $348.5(\mathrm{TO})$ & $348(\mathrm{TO})$ & $330(\mathrm{TO})$ \\
$363.1(\mathrm{TO} 2)$ & & & & \\
& $562.1(\mathrm{LO})$ & $545.5(\mathrm{LO})$ & $524(\mathrm{LO})$ & \\
\hline \hline
\end{tabular}

tions. In Ref. 52 also a detailed calculation of the twophonon density of states has been provided. Finally, the lattice dynamics of $\mathrm{CoO}$ has recently been calculated from first principles. $^{58}$

The four-parameter fit at room temperature also yielded the parameters $\varepsilon_{s}=14.0$ and $\varepsilon_{\infty}=5.0$, where the highfrequency dielectric constant has been deduced from fits of the reflectivity up to $2000 \mathrm{~cm}^{-1}$ (see, e.g., Fig. 4). The static dielectric constant compares well to our dielectric result, $\varepsilon_{s}$ $=14.2$, or to literature, where a value of 12.9 (Ref. 39) is reported. It is important to note that in the four-parameter fit, the Lyddane-Sachs Teller relation is automatically fulfilled and the static dielectric constant follows from the dielectric strength of the observed phonon mode. It seems that specifically the dielectric strength and concomitantly the longitudinal optical phonon frequency is at odds with the values reported in literature. ${ }^{55}$ From the dielectric strength the ionic plasma frequency can be directly calculated. Assuming ideal ionic bonding with valences of $Z= \pm 2$, we expect an ionic plasma frequency of $2040 \mathrm{~cm}^{-1}$ [see Eq. (6)]. This has to be compared with the experimentally observed room- temperature value of the ionic plasma frequency which amounts to $\Omega=987 \mathrm{~cm}^{-1}$. This value is very close to the one observed in $\mathrm{MnO}$, where $\Omega=1077 \mathrm{~cm}^{-1}$ has been determined. ${ }^{8}$ It indicates strong covalent contributions to the bonding in CoO. Specifically, the effective valence is found to be $Z^{*}=1.0$, much lower than the ideal ionic valence of $Z$ $=2$. From $\gamma$-ray diffraction it has been concluded that the $\mathrm{Co}-\mathrm{O}$ interaction is purely ionic. ${ }^{54}$

\section{B. Electronic excitations}

\section{Splittings of the $\mathrm{Co}^{2+}$ ground state}

Now, we want to turn to the additional excitation features that are visible in Figs. 4 and 5. The frequencies derived from our reflectivity measurements are listed in Table II and compared to excitation energies observed in FIR transmission, Raman, and neutron-scattering experiments. Evidently, many correspondences between our excitation frequencies and the ones in literature can be found. Note that we do not make any assignments of the origin of the excitations observed by these other techniques because in some studies additional features compared to our results have been observed. However, it is reasonable to assume that the excitations observed by these authors which coincide with our frequencies can be attributed to the same origin. Before we discuss in detail the approaches suggested to describe these excitations, we want to address the fact that a first distinction between the $Q_{1}-Q_{3}$ and the $M_{1}-M_{4}$ excitations can directly be made from the reflectivity data. Looking at Eq. (1) one can see that a contribution to the magnetic permeability $\mu(\omega)$ like, for example, magnetic-dipole (MD) transitions can lead to a reduction in the reflectivity and may produce a diplike feature in the spectrum. MD transition between $d$ states are symmetry allowed but usually are very weak with an oscil-

TABLE II. Electric quadrupole $\left(Q_{i}\right)$ and magnetic-dipole $\left(M_{i}\right)$ excitations in $\mathrm{CoO}$ observed in the present work. There are listed also excitation energies reported in previous far-infrared absorption, Raman, and neutron-scattering investigations together with the temperatures at which these experiments were performed at. All eigenfrequencies are given in $\mathrm{cm}^{-1}$.

\begin{tabular}{|c|c|c|c|c|c|c|c|c|}
\hline \multicolumn{4}{|c|}{ FIR } & \multirow{2}{*}{\multicolumn{2}{|c|}{ Raman }} & \multirow{2}{*}{\multicolumn{3}{|c|}{ Neutron scattering }} \\
\hline Reflectivity & \multicolumn{3}{|c|}{ Transmission } & & & & & \\
\hline (This work) & Ref. 30 & Ref. 31 & Ref. 32 & Ref. 53 & Ref. 59 & Ref. 56 & Ref. 60 & Ref. 61 \\
\hline $8 \mathrm{~K}$ & $2 \mathrm{~K}$ & $4.2 \mathrm{~K}$ & $10 \mathrm{~K}$ & $20 \mathrm{~K}$ & $10 \mathrm{~K}$ & $110 \mathrm{~K}$ & $10 \mathrm{~K}$ & $6 \mathrm{~K},\left(\begin{array}{lll}1.5 & 1.5 & 0.5\end{array}\right)$ \\
\hline \multirow[t]{2}{*}{$\sim 142\left(M_{1}\right)$} & 142.3 & & 146 & 143 & 143 & $145-178$ & & 163 \\
\hline & 146.5 & & & 148 & & & & \\
\hline $220\left(Q_{1}\right)$ & & 216 & 215 & & & $214-245$ & 218 & \\
\hline \multirow[t]{3}{*}{$221\left(M_{2}\right)$} & & 221 & 221.5 & 221 & 221 & & & 216 \\
\hline & & & 233 & & & & & \\
\hline & & & 243 & & & & & \\
\hline \multirow[t]{2}{*}{$250\left(M_{3}\right)$} & & 248 & 250 & & & & & 253 \\
\hline & & & 260 & & & & & \\
\hline $295\left(M_{4}\right)$ & & & 296 & 296 & 296 & $340-350$ & 313 & 315 \\
\hline $600\left(Q_{2}\right)$ & & & & & $530(?)$ & & & \\
\hline $680\left(Q_{3}\right)$ & & & & & & & & \\
\hline
\end{tabular}


lator strength of $10^{-6}$ and their contribution to $\mu(\omega)$ does not show up in optical spectra. The situation changes, however, when AFM order sets in and $\mu(\omega)$ reaches values which become comparable to $\varepsilon(\omega)$. The transitions $M_{1}-M_{4}$ fit well to such a scenario, showing diplike features in the reflectivity and appearing only below a temperature of about $100 \mathrm{~K}$, deeply in the AFM phase. Therefore, we assign these features to MD transitions. Note that similar observations of MD transition have been reported by Häusler et al. ${ }^{62}$ for the related compound $\mathrm{CoF}_{2}$, where $\mathrm{Co}$ has the same electronic configuration as in $\mathrm{CoO}$. While $M_{1}-M_{4}$ only appear far below $T_{N}$, the peaklike transitions $Q_{1}-Q_{3}$ do not show any significant changes at $T_{N}$ and are, obviously, not related to $\mu(\omega)$ but contribute to $\varepsilon(\omega)$. It is clear that on-site electricdipole transitions between the Co $d$-states are parity forbidden but can become allowed when the mixing with phonons breaks the inversion symmetry. Such a mechanism, however, should result in a characteristic temperature dependence, ${ }^{29,63-65}$ which is not observed. Therefore, we assign the transition $Q_{1}-Q_{3}$ to electric-quadrupole transitions, which are symmetry allowed with an expected oscillator strength of about $10^{-6}$, the same order of magnitude as the MD transitions.

Let us now turn to the expected level scheme (see Fig. 8) for $\mathrm{Co}^{2+}$ ions in $\mathrm{CoO}$. Following Liehr, ${ }^{66}$ who described the three-electron-hole cubic ligand-field spectrum in full detail, we can assign the lower two eigenstates, at 220 and $600 \mathrm{~cm}^{-1}$, to transitions between the ${ }^{4} F_{9 / 2}$ ground state which is split by the spin-orbit coupling. The energy level at $680 \mathrm{~cm}^{-1}$ obviously arises from a transition of the ground state to an excited one which is derived from the ${ }^{4} F_{7 / 2}$ state. Liehr ${ }^{66}$ deduced his results taking into account SOC in the atomic limit and then switching on the crystal field. Usually, the crystal-field splitting is calculated from the atomic limit and then SOC is introduced. In this case and assuming only the lowest crystal-field components, the crystal-field ground state splits into three levels only, the two highest levels being degenerate. Having made these qualitative assignments, we will now substantiate our conclusions by analyzing the energy-level scheme of the high-spin $\mathrm{Co}^{2+}$ ion following the approach by Sakurai et al. ${ }^{56}$ To describe the energy splittings of the ground state above $T_{N}$ we will take into account only SOC, while in the AFM phase an additional tetragonal crystal field and the effects of exchange splitting have be considered. Hence, our starting Hamiltonian for the ground state is given by

$$
H=\lambda(\mathbf{S L})+D\left[3 L_{z}^{2}-L(L+1)\right]+6 J\langle\boldsymbol{\alpha} \mathbf{S}\rangle(\boldsymbol{\alpha} \mathbf{S}),
$$

where $\lambda$ denotes the SOC constant, $D$ is the tetragonal $\mathrm{CF}$ parameter, and the last term is the nearest-neighbor exchange coupling. We want to recall some features of the superexchange interaction of $\mathrm{Co}^{2+}$ ions via oxygen in Co-O-Co fragments (for short we are using the hole representation $t_{2 g} e_{g}^{2}$ ). From a general point of view, one can distinguish the following exchange parameters: $J_{e e}$ between $e_{g}^{2}$ subshells, $J_{t t}$ between $t_{2 g}$ holes, and $J_{t e}$ between $e_{g}^{2}$ and $t_{2 g}$ subsystems (this statement is based on the internal symmetry of the exchange Hamiltonian; for details see Ref. 67). Note that the $e_{g}^{2}$ subshells are half filled and that therefore the effective orbital momentum vanishes. Hence, the leading term in the superexchange interaction of the Co ions can be written in the Heisenberg form in first approximation, i.e., $H_{a b}^{\mathrm{ex}} \approx J\left(\mathbf{S}_{a} \mathbf{S}_{b}\right)$, where $\mathbf{S}_{a}$ and $\mathbf{S}_{b}$ are the total spins of the $e_{g}^{2}$ subshell of the ion at site $a$ and $b$, respectively. The exchange parameters were constrained to one $J$ since $J_{e e}$ is dominating. Furthermore, it is possible to introduce an effective orbital momentum $\mathbf{L}$ with $L=1$ for the $\mathrm{Co}^{2+}\left({ }^{4} \Gamma\right)$ state as well as an effective total momentum $\mathbf{j}=\mathbf{L}+\mathbf{S}$ (see Refs. 41 and 56). Using the wave functions $\left|j, m_{j}\right\rangle$ listed in Ref. 41 , where $m_{j}$ is the quantum number of the component of $\mathbf{j}$ along the axis of quantization, it is straightforward to deduce the energy spectrum $\epsilon\left(j, m_{j}\right)$ of the $\mathrm{Co}^{2+}\left({ }^{4} \Gamma\right)$ state in crystal and molecular exchange fields

$$
\begin{gathered}
\epsilon\left(\frac{1}{2}, \pm \frac{1}{2}\right)= \pm \frac{25}{6} J, \\
\epsilon\left(\frac{3}{2}, \pm \frac{3}{2}\right)=\frac{3}{2} \lambda \pm \frac{11}{2} J-\frac{4}{5} D, \\
\epsilon\left(\frac{3}{2}, \pm \frac{1}{2}\right)=\frac{3}{2} \lambda \pm \frac{11}{6} J+\frac{4}{5} D, \\
\epsilon\left(\frac{5}{2}, \pm \frac{5}{2}\right)=4 \lambda \pm \frac{15}{2} J+D, \\
\epsilon\left(\frac{5}{2}, \pm \frac{3}{2}\right)=4 \lambda \pm \frac{9}{2} J-\frac{1}{5} D, \\
\epsilon\left(\frac{5}{2}, \pm \frac{1}{2}\right)=4 \lambda \pm \frac{3}{2} J-\frac{4}{5} D .
\end{gathered}
$$

These analytical expressions are very useful as a starting point for calculations, however, they have to be corrected since the axis of magnetization in $\mathrm{CoO}$ is not parallel to the tetragonal one. This leads to the last term in Eq. (9). To date, the values $\boldsymbol{\alpha}$ are still under debate (see Ref. 28), however, in most publications ${ }^{31,59}$ it is believed that the spins point into the $[\overline{1} \overline{17}]$ direction. Hence we set $\boldsymbol{\alpha}=\frac{1}{\sqrt{51}}(-1,-1,7)$. The experimentally observed magnetic-dipole transitions listed in Table II and located at 142, 221, 249, and $295 \mathrm{~cm}^{-1}$ were fitted using Eq. (9) with the SOC parameter $\lambda$, the crystalfield parameter $D$ and the magnetic exchange $J$ as free parameters. In these calculations we assumed that only transitions from the ground state $\epsilon(1 / 2,-1 / 2)$ were observed and that we detect all possible transitions with $\Delta m_{j}=0, \pm 1$. The best fit resulted in a levels scheme $\epsilon(1 / 2,-1 / 2)=0 \mathrm{~cm}^{-1}$, $\epsilon(1 / 2,+1 / 2)=145.7 \mathrm{~cm}^{-1}, \quad \epsilon(3 / 2,-3 / 2)=221.9 \mathrm{~cm}^{-1}$, $\epsilon(3 / 2,-1 / 2)=248.8 \mathrm{~cm}^{-1}$, and $\epsilon(3 / 2,+1 / 2)=294.3 \mathrm{~cm}^{-1}$ which is shown in Fig. 8. This calculated level scheme is very close to the experimentally observed magnetic excitations and resulted in parameters $\lambda=151.1 \mathrm{~cm}^{-1}, J$ $=17.5 \mathrm{~cm}^{-1}$, and $D=-47.8 \mathrm{~cm}^{-1}$. The transition to the level $\epsilon(3 / 2,+3 / 2)=432.9 \mathrm{~cm}^{-1}$ is magnetic dipole forbidden and, therefore, cannot be observed in our experiment.

Having derived the splitting parameters in the AFM state by fitting the energies of the MD transitions, we have to 


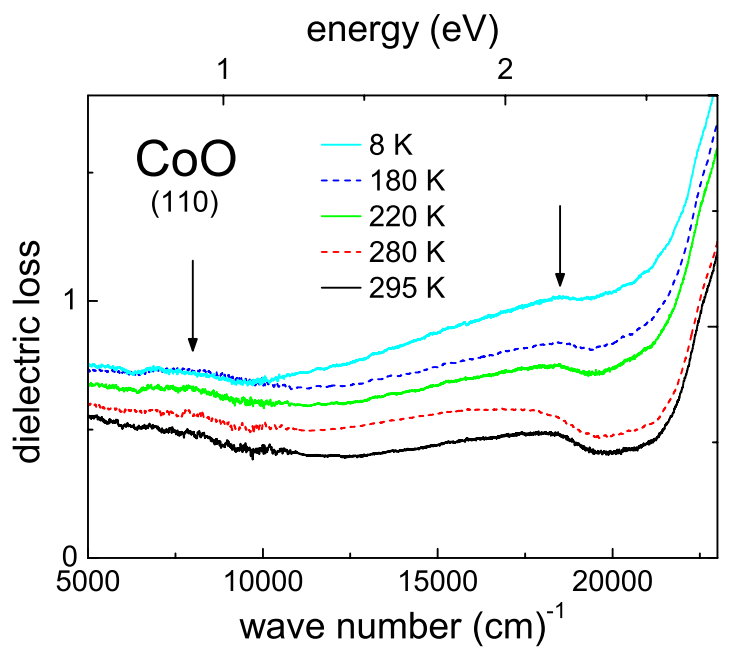

FIG. 7. (Color online) Dielectric loss vs wave numbers for $\mathrm{CoO}$ between 5000 and $23000 \mathrm{~cm}^{-1}$. Electronic $d$ - $d$ excitations are indicated by arrows.

compare these parameters to the energies of the transitions $Q_{1}-Q_{3}$ which are also present above the AFM transitions. We identify these transitions as electric-quadrupole transitions between the spin-orbit split crystal-field states. Note that transitions from the ground to the excited states with effective moment $j=5 / 2 \Leftrightarrow \Gamma_{7}, \Gamma_{8}$ are allowed only via electric-quadrupole mechanisms. This explains why the optical transitions near 600 and $680 \mathrm{~cm}^{-1}$ are not sensitive to the change in $\mu(\omega)$ upon the phase transition into the antiferromagnetic state. As can be seen from Eq. (10) by assuming $J=D=0$ the level separation between the ground state and the first-excited state above $T_{N}$ is given by $3 / 2 \lambda$. In $\mathrm{CoO}$ this immediately results in a SOC constant $\lambda=146.7 \mathrm{~cm}^{-1}$, in good agreement with the SOC parameter derived from the MD transition at low temperatures. To handle the slight discrepancies between calculation and observed energies, a detailed inspection of the corrections due to $t_{2 g}-e_{g}^{2}$ and $t_{2 g}$ $-t_{2 g}$ exchange interactions and the orthorhombic crystal field is necessary, which is out of the scope of the present paper.

\section{Higher-lying CF excitations}

Higher-lying CF excitations have been determined from optical absorption by Pratt and Coelho. ${ }^{29}$ They found two prominent absorption lines close to 8000 and $18500 \mathrm{~cm}^{-1}$. At low temperatures the high-energy transition revealed some substructure. These transitions were identified as transitions from the ${ }^{4} F_{1}$ ground state to the ${ }^{4} F_{2}$ and ${ }^{4} F_{1}$ excited states, which results in a crystal-field parameter of approximately $D q=900 \mathrm{~cm}^{-1}$. Our results are shown in Fig. 7. In the frequency-dependent dielectric loss as determined from the reflectivity, these transitions can only be detected by a close inspection of our spectra. Slight maxima, which are almost temperature independent and show no significant changes at the magnetic phase transition, appear close to 8000 and $18500 \mathrm{~cm}^{-1}$. Beyond $21000 \mathrm{~cm}^{-1}(=2.6 \mathrm{eV})$ the dielectric loss strongly increases, entering the frequency regime of strong absorption. The crystal-field excitations are broad and very weak in intensity and do not show any de- tectable splittings due to SOC effects. It is clear that these $d-d$ excitations are parity forbidden and gain intensity only via hybridization with other electronic orbitals or via coupling to phonons.

It is interesting to note that the optical gap as determined from the transport measurements rather coincides with the lower band edge of the $18500 \mathrm{~cm}^{-1}$ transition, which can be located close to $12000 \mathrm{~cm}^{-1} \sim 1.5 \mathrm{eV}$. It is however unclear how this on-site excitation can contribute to the dc hopping transport. The most plausible explanation of the transport gap certainly is the formation of an impurity band due to defects.

\section{SUMMARY AND CONCLUDING REMARKS}

We performed a careful characterization of singlecrystalline $\mathrm{CoO}$, utilizing magnetic and dielectric susceptibility as well as thermodynamic measurements. We determined the phase transition into the AFM state as $T_{N}=289 \mathrm{~K}$. The low-temperature heat capacity can well be described by a $T^{3}$ law with a Debye temperature $\Theta_{D}=451 \mathrm{~K}$. It seems that at low temperatures magnetic excitations play a minor role and do not contribute to $c_{p}$. This can be understood from the electronic excitations with a series of levels between 150 and $300 \mathrm{~cm}^{-1}$ at low $T$. It documents that SOC is strong compared to the magnetic exchange. From dielectric spectroscopy we determined $\varepsilon_{s}=14.2$ and an electronic gap from the dc transport $E_{g}=1.5 \mathrm{eV}$.

We analyzed the phonon dynamics including the spinphonon coupling at the antiferromagnetic ordering temperature. Damping and eigenfrequencies can be described by a normal anharmonic behavior. However, below $T_{N}$ a second mode splits off. The splitting amounts to $15.0 \mathrm{~cm}^{-1}$ at low temperatures, considerably lower than in the case of the isostructural antiferromagnet $\mathrm{MnO}$. This behavior might be explained taking the strong frustration of $\mathrm{MnO}$ into account, while in $\mathrm{CoO}$ Néel temperature and Curie-Weiss temperature are of the same order of magnitude. The ionic plasma frequency amounts to about $980 \mathrm{~cm}^{-1}$, signaling considerable covalent bonding.

In the second part of this work we determined the electronic and magnetic transitions. The measured reflectivity allows to discriminate between electric and magnetic-dipole transitions. The complete level scheme of the electronic excitation spectrum of $\mathrm{CoO}$ is plotted in Fig. 8. Starting from the electronic levels we determined two crystal-field excitations close to 8000 and $18500 \mathrm{~cm}^{-1}$. From these excitations we determine a crystal-field parameter $D q=900 \mathrm{~cm}^{-1}$, in good agreement with values reported from infrared absorption. ${ }^{29}$ These transitions are spin allowed transitions where only one electron from the $t_{2 g}$ ground state $\left(t_{2 g}^{5} e_{g}^{2}\right)$ is excited into an $e_{g}$ level $\left(t_{2 g}^{4} e_{g}^{3}\right)$. In CoO SOC effects are strong and have to be taken into account. We determined the splitting of the crystal-field ground state by SOC and identified three excited levels. From the separation of the first two levels we determine the SOC constant $\lambda=146.7 \mathrm{~cm}^{-1}$. This value is reasonable compared to the free ion case where $\lambda_{\text {Ion }}=176 \mathrm{~cm}^{-1} .{ }^{41}$ Due to the tetragonal distortion, $\Gamma_{7}$ and $\Gamma_{8}$ are not degenerate but are separated by $80 \mathrm{~cm}^{-1}$. In the free 


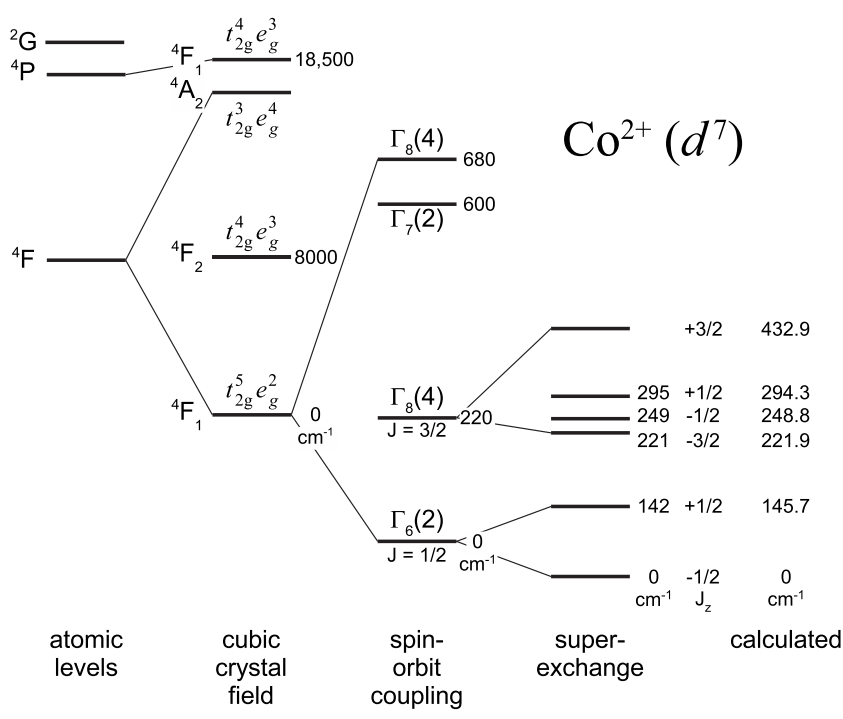

FIG. 8. Schematic splitting of the energy levels of the $\mathrm{Co}^{2+}$ ion in $\mathrm{CoO}$. The energies are given in $\mathrm{cm}^{-1}$ as determined in the present work. The magnetic-dipole transitions only appear below $100 \mathrm{~K}$, deep in the magnetically ordered phase. Note that different energy scales are used to display the effects of different interactions.

ion case these levels are degenerate and the separation from the ground state amounts to $4 \lambda$. Hence in the atomic limit we would expect one excitation at $606 \mathrm{~cm}^{-1}$, instead of two excitations at 600 and $680 \mathrm{~cm}^{-1}$, which we identified in
$\mathrm{CoO}$, in a crystal with strong covalent bonding. Finally, we determined a series of magnetic-dipole excitations at 142, 221, 249, and $295 \mathrm{~cm}^{-1}$, which can clearly be identified as magnetic-dipole transitions between the spin-orbit split crystal field levels whose degeneracy is completely lifted by molecular exchange fields in the AFM state. The levels for the lowest doublet and the first-excited quartet are also indicted in Fig. 8. These transitions appear at low temperatures $(<100 \mathrm{~K})$, are very weak, and exhibit almost no temperature dependence. We were able to describe the experimentally observed level scheme convincingly by a model taking the SOC parameter $\lambda=151.1 \mathrm{~cm}^{-1}$, the magnetic exchange $J=17.5 \mathrm{~cm}^{-1}$, and the tetragonal crystal-field parameter $D=-47.8 \mathrm{~cm}^{-1}$ into account. The calculated energy levels are also displayed in Fig. 8.

In conclusion, we were able to determine the complete phonon, electronic, and magnetic excitation spectrum of $\mathrm{CoO}$ within the charge-transfer gap by infrared spectroscopy. $\mathrm{CoO}$ is a prototypical and well-known example for a strongly correlated system, but its phononic, electronic, and magnetic excitation schemes offer an astonishing complexity.

\section{ACKNOWLEDGMENTS}

This research has partly been supported by the Deutsche Forschungsgemeinschaft DFG, via the Collaborative Research Center SFB 484 (University of Augsburg).
${ }^{1}$ A. Gorschlüter and H. Merz, Phys. Rev. B 49, 17293 (1994).

${ }^{2}$ B. C. Larson, J. Z. Tischler, W. Ku, C. C. Lee, O. D. Restrepo, A. G. Eguiluz, P. Zschack, and K. D. Finkelstein, Phys. Rev. Lett. 99, 026401 (2007).

${ }^{3}$ M. W. Haverkort, A. Tanaka, L. H. Tjeng, and G. A. Sawatzky, Phys. Rev. Lett. 99, 257401 (2007).

${ }^{4}$ J. Zaanen, G. A. Sawatzky, and J. W. Allen, Phys. Rev. Lett. 55, 418 (1985).

${ }^{5}$ S. Massidda, M. Posternak, A. Baldereschi, and R. Resta, Phys. Rev. Lett. 82, 430 (1999).

${ }^{6}$ W. D. Luo, P. H. Zhang, and M. L. Cohen, Solid State Commun. 142, 504 (2007).

${ }^{7}$ E. M. L. Chung, D. M. Paul, G. Balakrishnan, M. R. Lees, A. Ivanov, and M. Yethiraj, Phys. Rev. B 68, 140406(R) (2003).

${ }^{8}$ T. Rudolf, Ch. Kant, F. Mayr, and A. Loidl, Phys. Rev. B 77, 024421 (2008).

${ }^{9}$ A. B. Sushkov, O. Tchernyshyov, W. Ratcliff II, S. W. Cheong, and H. D. Drew, Phys. Rev. Lett. 94, 137202 (2005).

${ }^{10}$ T. Rudolf, Ch. Kant, F. Mayr, J. Hemberger, V. Tsurkan, and A. Loidl, Phys. Rev. B 75, 052410 (2007); J. Hemberger, T. Rudolf, H.-A. Krug von Nidda, F. Mayr, A. Pimenov, V. Tsurkan, and A. Loidl, Phys. Rev. Lett. 97, 087204 (2006); T. Rudolf, Ch. Kant, F. Mayr, J. Hemberger, V. Tsurkan, and A. Loidl, New J. Phys. 9, 76 (2007); J. Hemberger, H.-A. K. von Nidda, V. Tsurkan, and A. Loidl, Phys. Rev. Lett. 98, 147203 (2007).

${ }^{11}$ R. Valdes Aguilar, A. B. Sushkov, Y. J. Choi, S. W. Cheong, and H. D. Drew, Phys. Rev. B 77, 092412 (2008).
${ }^{12}$ Y. Yamashita and K. Ueda, Phys. Rev. Lett. 85, 4960 (2000).

${ }^{13}$ O. Tchernyshyov, R. Moessner, and S. L. Sondhi, Phys. Rev. Lett. 88, 067203 (2002); Phys. Rev. B 66, 064403 (2002).

${ }^{14}$ W. Klemm and W. Schüth, Z. Anorg. Allg. Chem. 210, 33 (1933).

${ }^{15}$ C. Henry La Blanchetais, J. Phys. Radium 12, 765 (1951).

${ }^{16}$ J. R. Singer, Phys. Rev. 104, 929 (1956).

${ }^{17}$ T. R. McGuire and W. A. Crapo, J. Appl. Phys. 33, 1291 (1962).

${ }^{18}$ E. Uchida, T. Nagamiya, H. Kondoh, Y. Nakazumi, T. Takeda, and N. Fukuoka, J. Phys. Soc. Jpn. 19, 2088 (1964).

${ }^{19}$ P. S. Silinsky and M. S. Seehra, Phys. Rev. B 24, 419 (1981).

${ }^{20}$ N. C. Tombs and H. P. Rooksby, Nature (London) 165, 442 (1950).

${ }^{21}$ S. Saito, K. Nakahigashi, and Y. Shimomura, J. Phys. Soc. Jpn. 21, 850 (1966).

${ }^{22}$ W. Jauch, M. Reehuis, H. J. Bleif, F. Kubanek, and P. Pattison, Phys. Rev. B 64, 052102 (2001).

${ }^{23}$ C. G. Shull, W. A. Strauser, and E. O. Wollan, Phys. Rev. 83, 333 (1951).

${ }^{24}$ W. L. Roth, Phys. Rev. 110, 1333 (1958).

${ }^{25}$ W. L. Roth, Phys. Rev. 111, 772 (1958).

${ }^{26}$ B. van Laar, Phys. Rev. 138, A584 (1965).

${ }^{27}$ D. Herrmann-Ronzaud, P. Burlet, and J. Rossat-Mignod, J. Phys. C 11, 2123 (1978).

${ }^{28}$ E. Ressouche, N. Kernavanois, L.-P. Regnault, and J.-Y. Henry, Physica B (Amsterdam) 385-386, 394 (2006).

${ }^{29}$ G. W. Pratt and R. Coelho, Phys. Rev. 116, 281 (1959). 
${ }^{30}$ R. C. Milward, Phys. Lett. 16, 244 (1965).

${ }^{31}$ M. R. Daniel and A. P. Cracknel, Phys. Rev. 177, 932 (1969).

${ }^{32}$ I. G. Austin and E. S. Garbett, J. Phys. C 3, 1605 (1970).

${ }^{33}$ U. Schneider, P. Lunkenheimer, A. Pimenov, R. Brand, and A. Loidl, Ferroelectrics 249, 89 (2001).

${ }^{34}$ M. B. Salamon, Phys. Rev. B 2, 214 (1970); M. B. Salamon, P. R. Garnier, B. Golding, and E. Buehler, J. Phys. Chem. Solids 35, 851 (1974).

${ }^{35}$ M. Massot, A. Oleaga, A. Salazar, D. Prabhakaran, M. Martin, P. Berthet, and G. Dhalenne, Phys. Rev. B 77, 134438 (2008).

${ }^{36}$ E. G. King, J. Am. Chem. Soc. 79, 2399 (1957); E. G. King and A. U. Christensen, ibid. 80, 1800 (1958).

${ }^{37}$ H. Watanabe, Thermochim. Acta 218, 365 (1993).

${ }^{38}$ E. N. Abarra, K. Takano, F. Hellman, and A. E. Berkowitz, Phys. Rev. Lett. 77, 3451 (1996).

${ }^{39}$ K. V. Rao and A. Smakula, J. Appl. Phys. 36, 2031 (1965).

${ }^{40}$ N. Tristan, V. Zestrea, G. Behr, R. Klingeler, B. Buchner, H. A. Krug von Nidda, A. Loidl, and V. Tsurkan, Phys. Rev. B 77, 094412 (2008).

${ }^{41}$ A. Abragam and B. Bleaney, Electron Paramagnetic Resonance of Transition Ions (Oxford University Press, New York, 1970).

${ }^{42}$ R. J. Radwanski and Z. Ropka, Physica B (Amsterdam) 345, 107 (2004).

${ }^{43}$ M. S. Kushwaha, Physica B \& C 112, 232 (1982).

${ }^{44}$ G. Assayag and H. Bizette, Compt. Rend. 239, 238 (1954).

${ }^{45}$ S. R. Elliott, Adv. Phys. 36, 135 (1987).

${ }^{46}$ P. Lunkenheimer, V. Bobnar, A. V. Pronin, A. I. Ritus, A. A. Volkov, and A. Loidl, Phys. Rev. B 66, 052105 (2002).

${ }^{47}$ P. Lunkenheimer, M. Resch, A. Loidl, and Y. Hidaka, Phys. Rev. Lett. 69, 498 (1992); P. Lunkenheimer, A. Loidl, C. R. Ottermann, and K. Bange, Phys. Rev. B 44, 5927 (1991); A. Seeger, P. Lunkenheimer, J. Hemberger, A. A. Mukhin, V. Y. Ivanov, A. M. Balbashov, and A. Loidl, J. Phys.: Condens. Matter 11, 3273 (1999); P. Lunkenheimer and A. Loidl, Phys. Rev. Lett. 91,
207601 (2003).

${ }^{48}$ F. Gervais and B. Piriou, Phys. Rev. B 10, 1642 (1974).

${ }^{49}$ J. F. Scott, Phys. Rev. B 4, 1360 (1971).

${ }^{50}$ A. Kuzmenko, REFFIT, University of Geneva, http:// optics.unige.ch/alexey/reffit.html

${ }^{51}$ R. A. Cowley, Adv. Phys. 12, 421 (1963).

${ }^{52}$ K. S. Upadhyaya and R. K. Singh, J. Phys. Chem. Solids 35, 1175 (1974).

${ }^{53}$ R. R. Hayes and C. H. Perry, Solid State Commun. 14, 173 (1974).

${ }^{54}$ W. Jauch and M. Reehuis, Phys. Rev. B 65, 125111 (2002).

${ }^{55}$ P. J. Gielisse, J. N. Plendl, L. C. Mansur, R. Marshall, S. S. Mitra, R. Mykolajewycz, and A. Smakula, J. Appl. Phys. 36, 2446 (1965).

${ }^{56}$ J. Sakurai, W. J. L. Buyers, R. A. Cowley, and G. Dolling, Phys. Rev. 167, 510 (1968).

${ }^{57}$ B. R. K. Gupta and M. P. Verma, J. Phys. Chem. Solids 38, 929 (1977).

${ }^{58}$ U. D. Wdowik and K. Parlinski, Phys. Rev. B 75, 104306 (2007).

${ }^{59}$ H.-h. Chou and H. Y. Fan, Phys. Rev. B 13, 3924 (1976).

${ }^{60}$ K. Tomiyasu and S. Itoh, J. Phys. Soc. Jpn. 75, 084708 (2006).

${ }^{61}$ Z. Yamani, W. Buyers, R. Cowley, and D. Prabhakaran, Physica B (Amsterdam) 403, 1406 (2008).

${ }^{62}$ K. M. Häussler, A. Lehmeyer, and L. Merten, Phys. Status Solidi B 111, 513 (1982).

${ }^{63}$ Y. Tanabe and S. Sugano, J. Phys. Soc. Jpn. 9, 753 (1954).

${ }^{64}$ A. D. Liehr and C. J. Ballhausen, Phys. Rev. 106, 1161 (1957).

${ }^{65}$ J. Deisenhofer, I. Leonov, M. V. Eremin, Ch. Kant, P. Ghigna, F. Mayr, V. V. Iglamov, V. I. Anisimov, and D. van der Marel, Phys. Rev. Lett. 101, 157406 (2008).

${ }^{66}$ A. D. Liehr, J. Phys. Chem. 67, 1314 (1963).

${ }^{67}$ M. V. Eremin and Y. V. Rakitin, Phys. Status Solidi B 82, 221 (1977). 